\title{
Index
}

accent, 4

ACD (Austronesian Comparative Dictionary), 38

ACE (Asian Corpus of English), 731, 748-749

AEC (ASEAN Economic Community), 727

Afghan English dictionary, 220

agony and ecstasy of world Englishes, 9-10

agriculture, population and, $17-18$

ALT (Assistant Language Teachers), Japan, 92

alterglobalization, 709, 720

American English, Philippine English and, 195-196

American slang, Indian English, 259-260

Americanization, globalization and, 9-10

Anglophone, marginalized traditions, 794

Anglophone Asian literature, 787

imperialism and, 793

migration and, 793

racial mixture and, 793

Southeast Asia, 792-796

tiger symbol, 794

angloversals, 172-173 animal husbandry, population and, 17-18

APSC (ASEAN Political-Security

Community), 727

APT (ASEAN+3), 81-82

English in education, 91

monolingualism, 82-84

national language in education, 91

Arabic, 35-36

lexical borrowing in Southeast

Asia, 41

areoversals, 172-173

articles, irregular use, 176-177

ARWU (Academic Ranking of World Universities), 157-159

Aryan Vedic speakers, Rgveda and, 20

ASCC (ASEAN Socio-Cultural Community), 727

ASEAN (Association of Southeast Asian Nations), 27

AEC (ASEAN Economic

Community), 727

APSC (ASEAN Political-Security

Community), 727

ASCC (ASEAN Socio-Cultural

Community), 727

Charter of 2009, 728

globalization and, 707

The Handbook of Asian Englishes, First Edition. Edited by Kingsley Bolton, Werner Botha, and Andy Kirkpatrick.

(C) 2020 John Wiley \& Sons, Inc. Published 2020 by John Wiley \& Sons, Inc. 
ASEAN (Association of Southeast Asian Nations) (cont'd)

history of English, 727-731

internationalization of global education, 10

language policies, 726-727

members, 81

multilingualism and, 725-727

standardization and, 718-719

ASEAN ELF

community and, 735

cooperative atmosphere, 734

cultural values and, 735

identity expression and, 735

linguistic features, 731-735

sociocultural ELF, 731-735

Asian ELF, 731

first language influence on second, 732

Asian Englishes

Asian Englishes and Asian languages, 15-48

Asian Englishes and statistics, 49-80

Asian Englishes and language policies, 81-105

Asian Englishes and Asian schools, 107-131

Asian Englishes and Asian universities, 133-168

Asian Englishes and morphosyntax, 169-188

Asian Englishes and phonology, 189-207

Asian Englishes and lexicography, 209-240

Asian Englishes and globalization, 707-721

Asian Englishes and English as a Lingua Franca (ELF), 725-740

Asian Englishes and corpus linguistics, 741-762

Asian Englishes and popular culture, 763-786

Asian Englishes and Asian literatures, 787-811

Asian Englishes and Asian religions, 813-832
Asian Englishes and linguistic landscapes, 833-861

Asian Englishes and legal systems, 863-886

as field of study, 2

future study, $8-10$

Asian Englishes, 2

Asian Englishes: Beyond the canon

(Kachru), 3

Asian Games, 587

Asian legal systems. See legal systems

Asian literatures, 787-811

cultural nationalism, 796-798

diasporic writing, 791, 798-799

feminism, cultural nationalism and, 798

legitimacy, 790

Mo, Timothy, 791

nationalist authors, 788

nationalist canon, 791

postcolonial calibrations, 802

postcolonial studies and, 790-791

queer diaspora, 798-799

Roy, Arundhati, 791

Rushdi, Salman, 791

Southeast Asia, 792-796

Three Circles model, 802

transpacific, 799-801

world English literatures, 801-806

Asian popular culture, 763-786

development, 764-766

Expanding Circle Englishes, 767-768

Outer Circle Englishes, 766-767

Atharvaveda people, 21

Austroasiatic languages

Khasic, 18, 20

Kra-Dai languages and, 23

lexical borrowing, 37

MSEA, 23

Munda, 18, 20

Southeastern Riverine Hypothesis, 23

Tibeto-Burman languages and, 23

Vietnamese, 34

Austronesians, Papuan populations and, 30

Aw, Tash, 801, 802, 804 
Bangladesh, 51, 297-315

Bangla

code-switching, 306-308

creating writing, 305

education and, 301-302

media and entertainment, 303

nationalism and, 297-299

politics, 303-304

status, 300-301

Common Law, 867-869

East India Company and, 298

ELT (English language teaching), 108-109

EMI higher education, 138, 154

English in schools, 108-109

English users, 53-54

English-medium education system, 108-109

functions of English creative writing, 304-305

education, 301-302

electronic communication, 305

employment, 302-303

entertainment, 303

media, 303

NGOs (non-government organizations), 304

politics, 303-304

history of English, 298-299

languages spoken, 53

linguistic ecology, 299-300

status of English, 300-301

Bangladesh English dictionary, 221

Bangladeshi English, 297-315

code-mixing and, 306-308

code-switching and, 306-308

features, 306-308

linguistic carelessness, 309-311

nativization, 309

SMS texts, 306

sociolinguistics, 306-307

technology and, 306-308

varietal features versus errors, 309-310

Bhutan, 51

Common Law, 869-870

legal system English use, 869

Bhutan English dictionary, 221
Bible, English, 816

bilingual creativity, 1 popular culture and, 769-771

bilingual dictionaries, 210-211

bilingualism

instinct, 2

legal systems, 867-869

The black isle (Tan), 804-805

Book of Han, 27

BPO (business process outsourcing), 9-10 American slang, 259-260

The British Council, 50

British East India Company, 247-248

Brunei, 51, 399-418

Bahasa Indonesia, 413

Bahasa Melayu, 109

Common Law, 865

Dwibahasa, 412-413

Dwibahasa policy, 109-110

East India Company, 400

EMI higher education, 141-142, 154

English in Constitution, 400

English in schools, 109-110

English users, 54

functions of English, 402-403

history of English, 400-402

language in social media, 409-412

language policies, 402-403

language-in-education policies, 412-414

languages of, 399-400

legal system, monolingual, 865

linguistic landscape studies, 836

Melaya Islam Beraja, 400

official language, 83

phonology, 192

Brunei English, 399-418

discourse, 406-407

existence, attitudes toward, 408-409

features, 403-408

lexis, $407-408$

morphosyntax, 405-406

phonology, 403-405

Brunei English dictionary, 221

Buddhism

Sanskrit and, 33-34, 317-318

spread to Southeast and East Asia, 33-34 
Burma. See Myanmar

Butler English, 175

calibrations, 802

Cambodia, 51, 649-666

ASEAN and, 654

bilingual signage, 658-659

Civil Law, 872

dignitary visits, 659

education, foreign language, 652

EMI (English-medium instruction), 655 higher education, 146-147, 156

English in Cambodia, 649-666

English in schools, 116-117

English users, 59

history of English, 651-653

IFL (Institute of Foreign Languages), 653

Institute of Foreign Languages at the Royal University of Phnom

Penh, 116

Khmer Rouge, 651

linguistic landscape studies, 837

multilingualism, 649-650

official language, 83

UNTAC (United Nations Transitional

Authority for Cambodia), 651

Cambodian English

ACC (Accreditation Committee of Cambodia), 662

bilingual signage, 658-659

CamTESOL, 662

culture and, 658-660

features, 656-658

functions of, 654-655

future of, 663

grammar, 657

language education, 660-663

language policies, 660-663

lexis, 657

literature, 659-660

newspapers, 655

as official language, 654

phonology, 657

ratification, 656

status, 654-655

technology, 655

TV and radio, 659
Cambodian English dictionary, 221

Cambridge English, 50

Cantonese, 54

Macau, 530

capitalism, globalization and, 707

Catford, John C., 2

CEFRL (Common European Framework of Reference for Language), 118,697

CET (College English Test), 93

China, 51, 503-528

bilingual education, 505

business / trade, international, 510-511

EMI higher education, 149-150, 156

English in China, 503-528

English in Chinese schools, 117-118

English literacy, 505

English users, 59

international education, 509-510

language-in-education policies, 89

linguistic landscape studies, 837-838

minority languages, 60

Mongolian, 60

NCEE (National College Entrance

Examination), 508

phonology, 197-198

Putonghua, 507

script reform, 87

southern geography, 16

status of English, 507-508

Tibetan, 60

Uyghur, 60

writing system, 87

Zhuang, 60

China English dictionary, 221-222

Chinese English, 504

American English and Chinese English, 513-514

British English and Chinese English, 513-514

code-mixing/code-switching, 517-521

in communications, 512-513

CPE (Chinese Pidgin English), 541

Crazy English movement, 505

education, 505-507, 508-510

EMI (English-medium instruction), 509

functions, 508-513 
grammar, 516

lexical features, 515

linguistic features, 513-514

in media, 512-513

phonology, 515-516

pragmatic features, 517

Putonghua, 507

research on, 504-507

sociolinguistics, 507-508

status of, 507-508

tourism, international, 511-512

universities, 509

varieties, 181-182

Chinese languages, 34

Gan group, 60

Guanhua group, 60

Hakka speakers, 35

Han, 34

Hanfite Muslim Chinese community, 35

Kejia group, 60

Min group, 60

Min languages, 35

Singaporean speakers, 424

Sinitic, 34

standard, promotion, 507

Wu group, 60

Xiang group, 60

Yue group, 60

Christianity, English and, 818-820

Civil Law-based legal systems

Cambodia, 872

Indonesia, 872-873

Japan, 870-871

Laos, 872

Macau, 871

South Korea, 870-871

Thailand, $871-872$

Timor Leste, 873

Vietnam, 872

code-mixing

Bangladeshi English and, 306-308

Chinese English, 517-521

legal systems, 877-878

Nepali English, 328-329

Pinoylish, 495

Sri Lanka, 345-347

Taiwanese English, 559-560 code-shifting, legal systems, 877-878

code-switching

Chinese English, 517-521

legal systems, 877-878

Madarin-Hokkien (Taiwan), 549

Minglishes (Indian English) and, 267

Nepali English, 328-329

Pinoylish, 495

superdiversity and, 716-717

Collected works of B.B. Kachru, 1

colonial pluralism, 793

Philippines, 795

colonialism

European, 711

India, 248

Indonesia, 118, 607

Japanese in Korea, 586-587

lexicography and, 213-214

Myanmar, 360

Outer Circle Asian societies, 51

Philippines

American colonialism, 480-481

Spanish colonial period, 480

US colonialism, 114-115

postcolonial Englishes, 4

postcolonial nation-states, 792

postcolonial theory, linguistics and, 218-219

Sri Lankan language policies, 338-340

Thai English and, 632

Vietnam, French colonialism, 123,684

Common Law-based legal systems, 864-865

Bangladesh, 867-869

Bhutan, 869-870

Brunei, 865

Hong Kong, 867-869

India, 865-867

Malaysia, $867-869$

Maldives, 869-870

Myanmar, 869-870

Nepal, 869-870

Pakistan, 865-867

Philippines, 865-867

Singapore, 865

Sri Lanka, 867-869 
community law, 874-875

connections, globalization and, 709

contact languages

Arabic, 35-36

Chinese, 34-35

creoles, 31

Islamic invasions, 35-36

Malay, 34

Persian, 35-36

pidgins, 31

Sanskrit, 31-34

corpora

ACE (Asian Corpus of English), 748-749

CLARIN (Common Language Resources and Technology Infrastructure), 749

Diachronic Corpus of Hong Kong English, 754-756

Diachronic Corpus of Singapore English, 754-756

DIY (do-it-yourself), 748

GloWbE (Corpus of Global Web-based English), 747

ICE (International Corpus of English), 745-746

ICNALE (International Corpus Network of Asian Learners of English), 748-749

Kolhapur Corpus, 742, 745

LINDSEI (Louvian International Database of Spoken English Interlanguage), 747

NOW (News on the Web), 747

specialized, web and, 746-747

typology, 745-749

VOICE (Vienna-Oxford International

Corpus of English), 748

corpus linguistics, 741-762

analysis, 744

annotation, 743

compilation, 743

LOB (Lancaster/Oslo-Bergen)

Corpus, 743

corpus planning, 85

China, 87

Indonesia, 86-87
Japan, 87

Korea, 87

orthographic reform and, 86

Philippines NLI, 86

corpus revolution, 742

creoles, acquisition, 31

cross-border law

private law, 874

public law, 873-874

Crystal, David, 49, 51, 52

CSAT (College Scholastic Ability Test), 93

cultural homogenization, 708

cultural nationalism, Asian literatures and, 796-798

culture. See also Asian popular culture economic diversity, 765

religious practice, $764-765$

decorative English, 769-770

Diachronic Corpus of Hong Kong English, 754-756

Diachronic Corpus of Singapore English, 754-756

diasporic writing, Asian literatures, 798-799

dictionaries, 209-240

Afghan English, 220

Bangladesh English, 221

Bhutan English, 221

bilingual, 210-211

Brunei English, 221

Cambodian English, 221

China English, 221-222

early twentieth century, 211-215

global

Encarta world English dictionary, 230-231

Green's dictionary of slang, 233

Grolier international dictionary, 231

Lonely Planet travel guides, 231-232

Oxford English dictionary, 232-233

Hong Kong English, 222

Indian English, 223-224

Indonesian English, 224

Japanese English, 225

Korean English, 225 
Lao English, 225

LEME (Lexicon of Early Modern

English), 211

Macquarie dictionary, 217

Malaysian English, 225-226

methodology, 219-220

Mongolian English, 226

Myanmar English, 226

Nepal English, 226

nineteenth century, 211-215

Pakistan English, 226

Philippine English, 227

pre-nineteenth century, 210-211

Singapore English, 227-228

Sri Lankan English, 228-229

Taiwanese English, 229

Thai English, 229

Vietnamese English, 229

discourse particles, 184

disembedding, globalization and, 709

diversity, 715-716

economic diversity, 765

ethnic diversity, 764-765

language families, 725

superdiversity, 715-716, 835

divine languages, 817

DIY (do-it-yourself) corpora, 748

Dong Son culture, 27

Dravidian languages, 20

India, 246

Indo-Aryan speakers and, 29-30

East Asia, EMI higher education, 141-146

economic diversity, 765

Educated Indian English (EIE), 253

education

additional language teaching, 90-91

APT (ASEAN+3), 91

Education Testing Service, 50

EF (Education First), 50, 65

EMI (English-medium instruction)

global education and, 10

higher education, 136-157

English Proficiency Index, 50

internationalization, 10

language-in-education policies, 88-90 pedagogy policies, 92-93

teacher qualification policies, 91-92

Education Testing Service, 50

EF (Education First), 50, 65

English Proficiency Index, 50

EF English Proficiency Index, 65-69

Myanmar, 363

EFL (English as Foreign Language), 107

Expanding Circle, 107

ELT (English language teaching)

Bangladesh, 108-109

Thailand, 634

EMI (English-medium instruction), 7

Cambodia, 655

Chinese schools, 89, 509

global education and, 10

globalization and, 719

higher education

Asian region, 136-137

Expanding Circle contexts, 146-153, 155-157

global collaboration and, 134-136

Outer Circle contexts, 137-146, 153-155

Hong Kong, 462

promoting, international rankings and, 157-159

Thailand, 642

Vietnam, 688-689

"English as an Asian language"

(Kachru), 2

English Proficiency Index, 50

English studies, paradigm shift, 1-2

English Today, 2

English users, 52-53

Bangladesh, 53-54

Brunei, 54

Cambodia, 59

China, 59

Hong Kong, 54

India, 54-55

Indonesia, 61

Japan, 61

Laos, 62

Macau, 62

Malaysia, 55

Myanmar, 55-56 
English users (cont'd)

Nepal, 56

Pakistan, 56-57

Philippines, 57

Singapore, 57

South Korea, 62-63

Sri Lanka, 58

Taiwan, 63

Thailand, 63-64

Vietnam, 64

English World-Wide, 2

Englishes, postcolonial, 4

Englishes versus English, 3

English-medium education systems, Bangladesh, 108-109

ENL (English as Native Language), 107

ESL (English as a Second Language), 107

Outer Circle, 107

ethnic diversity, 764-765

ETS (Educational Testing Service), 65

European colonization, 711

eWAVE (World Atlas of Morphosyntactic

Variation in English), 172-173

Expanding Circle, 1, 5-6, 169, 713

EFL (English as Foreign Language), 107,171

EMI higher education, 155-157

East Asian societies, 149-153

Southeast Asian societies, 146-149

English in schools

Cambodia, 116-117

China, 117-118

Indonesia, 118-119

Japanese, 119

Laos, 120-121

Macao, 121

South Korea, 119-120

Taiwan, 121-122

Thailand, 122

Vietnam, 123

varieties

Chinese English, 181-182

Japanese English, 182

Korean English, 182

Thai English, 183
Expanding Circle Asian societies, 1-10, 503-703

Expanding Circle Englishes, 503-703

feminism, cultural nationalism and, 798

first language influence on second language, 732

Five star billionaire (Aw), 804

future study, 8-10

geography, morphosyntactic features and, 176

geosemiotics, 835

Ghosh, Amitav, 802, 803-804

The glass palace (Ghosh), 803-804

global education

EMI in higher education, 134-136

internationalization, 10

globalization, 707

alterglobalization, 709, 720

Americanization and, 9-10

ASEAN and, 707

Asian Englishes and

anecdotes, 710

disembedding, 711-715

reembedding, 711-715

connections, 709

cultural homogenization and, 708

dimensions, 709

disembedding, 709

EMI (English-medium instruction) and, 719

identity politics, 709

IMF (International Monetary Fund) and, 708

mixing, 709

mobility, 709

Nepali English, 325-326

phonology and, 201-202

risk, 720

speed, 709

standardization and, 718-719

World Bank and, 708

WTO (World Trade Organization) and, 708 
GloWbE (Corpus of Global Web-based English), 747

grammar, 4

grammaticity, ICE corpus, 176

Greene, Graham, 788

Hakka speakers, 35

Halliday, Michael A.K., 2

higher education, 133-168

Hinduism

Sanskrit and, 317-318

Vedas translations, 822

history of English, in India, 247-250

HKE (Hong Kong English), 176

HKSAR (Hong Kong Special

Administrative Region), proficiency measurement, 70

Hmong-Mien languages, MSEA, 23-24

homogenization, cultural, 708

Hong Kong, 51, 449-478

aided schools, 110

biliteracy, 457

Cantonese, 451-452

Common Law, 867-869

culture, 452-453

EMI higher education, 142-143, 154

English in schools, 110-112

English users, 54

government schools, 110

history of English, 449-451

HKSAR (Hong King Special

Administrative Region), 111

Hong Kong people, 452

internalization of English, 772

language planning, 456-458

language policies, 456-458

languages, 54, 454

Lennon walls, linguistic landscape study, 842-854

linguistic landscape studies, 837-838

multilingualism, 453-456

phonology, 192-193

private schools, 110

Putonghua, 451-452

SAR (Special Administrative Region), 843 sociolinguistic context, 451-453

status of major languages, 467

trilingualism, 111, 457

Hong Kong English, 449-478

accent, 468-469

community use, 463, 465-467

consonants, 468

diphthongs, 468

in education, 461-463

EMI (English-medium instruction), 462

features, 467-471

fricative system, 469

in government, 458-459

grammar, 470-471

Konghish, 471-473

in legal system, 459-461

LegCo, 458-459

in media, 465

official languages, 458

sociolinguistics, 177

varieties, $177-179$

vocabulary, 469-470

vowel contrasts, 468

workplace, 463-465

written languages at work, 464

Hong Kong English dictionaries, 222

IAL (international auxiliary language), 817

IAWE (International Association for World Englishes), 2

ICE (International Corpus of English), 171, 745-746

grammaticity, 176

ICNALE (International Corpus Network of Asian Learners of English), 748-749

identity, language and, 793-794

identity languages, 726

India, 246

linguistic stereotypes, 771-772

popular culture and, 771-772

identity politics, globalization and, 709

ideology, language and, 789, 793-794 
IDP (International Development Program), 50

IELTS (International English Language Testing System), 50, 510 proficiency levels, 65-69

IndE. See Indian English

India, 51, 243-277

Austro-Asiatic language family, 246 bilinguals, 253

BPO (business process outsourcing), American slang, 259-260

British East India Company, 247-248

Butler English, 175

Christian missionaries, 247

Dravidian languages, 246

EMI higher education, 138-139, 154

English as official language, 268

English in education cognitive development and, 268-269

Indian identity and, 270

minority languages and, 269-270

regional languages and, 268-269

second language acquisition and, 270-272

as subject, 268

tribal languages and, 269-270

English in schools, 112

English users, 54-55

functions of English

administration, 250-251

education, 251

imaginative, 250

instrumental, 250

law, 251

media, 252

regulative, 250

Hindustani English, 194

historical milestones, language and, 245

history of English in India, 247-250

class and, 248

colonial control and, 248

Convent English, 247

education and, 248-249

Three Language Formula, 249

identity languages, 246

Indo-European language family, 245
Indo-Aryan subfamily, 245

Indo-Iranian subfamily, 245

Kamada English, 194

language boundaries, 717

linguistic diversity, 245-246

linguistic landscape studies, 838

literary languages, 246

local language in legal system, 865-866

multilingualism, 245-247

NCERT (National Council for

Educational Research and

Training), 269

northeastern geography, 16

official languages, 246

phonology, 193-195

regional languages, 246

scheduled languages, 246

Sino-Tibetan language family, 246

social mobility, English and, 112

status of English, 250-252

superdiversity, 716

Three Language Formula, 249

three-language formula, 194

Tibeto-Burman language family, 246

trilingualism, 112

Indian English, 176, 243-277

academic debates, 270-272

versus British/American English, 270-272

creative writers, 244

culture and, 263

Educated Indian English (EIE), 253

features, 253-263

India Today, 245

lexicon, 256

American slang, 259-260

archaisms, 258

calques, 259

clichés, 258

clippings, 259

collocational differences, 258

diction, 257-258

idioms, 258

Indian expressions, 257

Indian words, 257

initialisms, 259

innovations, 258 
loanwords, 257

register crossover, 258

semantic shift, 259

linguistic area, 254

Minglishes

cultural significance, 266

domains, 263-266

linguistic theory, 267

morphology, 255-256

phonetics, 254-255

phonology, 254-255

reading, 244

spelling

place names, 261

pronunciations, 262

substrate influence, 253-254

subvarieties based on mother tongues, 262

syntax, 260-261

variation, acquisitional background and, 262

varieties, 178-179

Indian English dictionaries, 223-224

The Indianization of English: The English language in India (Kachru), 2

"The Indianness in Indian English" (Kachru), 2

indirect questions, inverted word order, 176

Indo-Aryan languages, 20

Dravidian speakers and, 29-30

Indo-Burmese Arc, Sino-Tibetan languages and, 24

Indo-Gangetic language, 29

Indonesia, 51, 605-627

Bahasa-Indonesia, 118

Balinese, 118

Batak, 118

Civil Law, 872-873

colonial era, 118, 607

corpus planning, 86-87

Dutch East Indies, 118, 608

EMI higher education, 147, 156

English in Indonesia, 605-627

English in schools, 61, 118-119

English users, 61 history of English, 607-608

indigenous languages, 118, 606

Javanese, 118

Language Agency (Badan Bahasa), 607

language loss, 606-607

languages, future, 622

major languages, 61

Minangkabau, 118

Ministry of Education and Culture, 607

multilingualism, 605-607

Pancasila, 605

regional languages, 606

religious heritage, 605

Sanskrit loan words, 39-40

Sundanese, 118

Indonesian English

cultural aspects, 613-615

education system, 616 materials for teaching, 621

policy, 617-619

sector composition, 616-617

subject coverage, 619-620

teacher evaluation, 621-622

teacher qualifications, 620

teaching, 620

error analysis, $612-613$

functions of, 608-609

future of, 622

language acquisition, 609-610

policy, 617-619

spoken English, 610-611

tourism, 613-614

written English, 611

Indonesian English dictionaries, 224

Indosphere, 27

Inner Circle, 107, 169, 713

ENL (English as Native Language), 107,171

WAMVE and, 174

internationalization

global education, 10

phonology and, 201-202

inversion

indirect questions, 176

main clause yes/no questions, 177

wh-questions, 177

Iron Age, Southeast Asia trading, 27 
ISEA (Island Southeast Asia)

Austronesian speakers, 26

geography, 16

Jatakas, 27

Niddesa, 27

Papuan speakers, 25-26

political integration, 26-28

Ramayana, 27

Srivijayan expeditions, 27

Islam

Brunei, 399

English and, 820-822

invasions, contact languages and, 35-36

Malay and, 34

Qur'an translations, 822

Japan, 51, 569-584

ALT (Assistant Language

Teachers), 92

American occupation, 571-572

Civil Law, 870-871

EMI higher education, 150-151, 156

English as foreign language, 773

English in Japan, 569-584

English in schools, 119

English Language Education

Associations, 572

English users, 61

history of English, 570-572

JET (Japan Exchange and Teaching) program, 92

linguistic landscape studies, 839

Meiji period, 571

monolingualism, 570

phonology, 198-199

script reform, 87-88

writing system, $87-88$

Japanese English

in education, $572,574-575$

features, 578-581

ideology, 570

influence, attitudes toward, 580-581

Japanese-made English (wasei eigo), 579

nihonjinron treatises, 573 as official language, 575-576

ornamentalism, 577-578

policies, 572-573

prospects, $576-577$

pseudo-loans, 579-580

TOEFL scores, 569

varieties, 182

words which come from outside, 578-579

workplace, 569

Japanese English dictionaries, 225

JET (Japan Exchange and Teaching) program, 92

JGSS (Japanese General Society Surveys), 61

Kachru, Braj B., 1, 2-3, 5, 9, 11, 54, 98, 107, 127-129, 169-171, 189, 198, 200, 217, 237, 244-245, 250, 253-254, 256-258, 263, 270-271, 323, 339-340, 350, 367, 402-403, 526, $553,585,634,636,638,641,651$, 685, 712-713, 724, 728-729, 738, 748, 766-767, 769, 772, 789-790, 801-802, 807-808, 813

The alchemy of English: The spread, functions, and models of non-native Englishes, 2

Asian Englishes: Beyond the canon, 3

"English as an Asian language," 2

The Indianization of English: The English language in India, 2

The other tongue: English across cultures, 2

World Englishes, 2

Kachru, Yamuna, 2, 245, 254, 263, 271, 273-276

Khasic, 18, 20

knowing a language, 49-50

Kolhapur Corpus, 742, 745

Konglish, 589

Korea, 51, 585-604

Asian Games, 587

attitudes toward English, 599-600

bilingualism, 586-587

Chinese characters, 586

English in Korea, 585-604 
English Villages, 598

globalization, English and, 597-599

history of English, 587

K-Beauty, 596

kirogi families, 598

Koreanized English, 589

language in, 586-587

linguistic landscape, 596-597

script reform, 87-88

South/North linguistic differences, 586

suppression of language, Japanese colonization and, 586-587

Korean English, 585-586

advertising and, 596

cultural aspects, 594-597

in education, 599-600

English officialization, 598-599

English Villages, 598

Englishization of Korean, 593-594

features, 588-594

grammar, 590-592

kirogi families, 598

Konglish, 589

K-Pop, 595

lexis, 592-593

music and, 595-596

phonology, 590

South Korea, 588

television and, 594-595

terms for, 588-589

varieties, 182

Korean English dictionaries, 225

Kra-Dai languages, 21-22

Austroasiatic languages and, 23

MSEA, 24-25

Sinitic influence, 37-38

Kurukh, Munda languages, 18

language contact, 171

language replacement, 29

language shift, 29

legal systems

code-mixing and, 877-878

code-shifting and, 877-878

code-switching and, 877-878

hybrid texts, 877

lexical borrowing, 876 lexical borrowing, 28

MSEA, 30

South Asia, 29-30

structural borrowing, 28-29

structural convergence, 28-29

Wallacea, 30

language dominance, 834

language families in South/Southeast Asia, 17-18

language in place, 834

language mixing, popular culture and, 769-771

language replacement, language contact and, 29

language shift, language contact and, 29

language-in-education policies, 88-90

Lao English dictionaries, 225

Laos, 51, 667-682

ASEAN, 674-677, 678

Civil Law, 872

education, 669-670, 672-674

EMI higher education, 147-148, 156

English in Laos, 667-682

English in schools, 120-121

English users, 62

French, 120-121

French in, 670-671

in government, 674-677

Latin script, 672-673

literacy in national language, 669-670

major languages, 61

MOE (Ministry of Education), 668

MOES (Ministry of Education and Sports), 668

multilingualism, 668-670

National Education System Reform Strategy, 673

private sector, 677-679

teacher training colleges, 677-678

legal systems in Asia, 863-886

bilingualism, 867-869

Civil Law-based

Cambodia, 872

Indonesia, 872-873

Japan, 870-871

Laos, 872

Macau, 871 
legal systems in Asia (cont'd)

South Korea, 870-871

Thailand, 871-872

Timor Leste, 873

Vietnam, 872

colonial expressions, 878-879

Common Law-based, 864-865

Bangladesh, 867-869

Bhutan, 869-870

Brunei, 865

Hong Kong, 867-869

India, 865-867

Malaysia, 867-869

Maldives, 869-870

Myanmar, 869-870

Nepal, 869-870

Pakistan, 865-867

Philippines, 865-867

Singapore, 865

Sri Lanka, 867-869

community law, 874-875

cross-border law

private law, 874

public law, 873-874

culturally specific terminology, 878-879

language contact, lexical borrowing and, 876

monolingual English law, 865

nativized grammar, $878-879$

religious law, 875

shariah law, 865, 875

LEME (Lexicon of Early Modern English), 211

Lennon walls, Hong Kong linguistic landscape study, 842-854

lexical borrowing. See also loanwords

Arabic to Southeast Asia, 41

Austroasiatic into Indo-Aryan, 36

influences, 40

language contact and, 28

MSEA (Mainland Southeast Asia) and, 37

lexicography, 209-240. See also dictionaries, Asian Englishes

codification, 217

colonial-era, 213-214
A descriptive dictionary of British Malaya, 213-214

Dictionary of American regional English, 217

Dictionary of the English/creole of Trinidad \& Tobago, 217

The English dialect dictionary, 217

Fifth report from the Select Committee on the affairs of the East India Company, 212

Hobson-Jobson, 213, 214-215

The Middle English dictionary, 217

naturalization, 215-216

non-standard language, 217-218

standard language, 217-218

lexis, 4, 209-240

Brunei English (BrunE), 407-408

Cambodian English, 657

Korean English, 592-593

Myanmar English, 367

Pakistani English, 284

Sri Lankan English, 345

Taiwanese English, 558-559

Thai English, 637-638

LINDSEI (Louvian International Database of Spoken English Interlanguage), 747

linguistic diversity, 715-716

linguistic elitism, 318

linguistic epicenters, 752-754

linguistic landscapes, 755-756, 801-806, 833-861

in Brunei, 836

in Cambodia, 837

in China, 837-838

in Hong Kong, 837-838

in India, 838

in Indonesia, 838-839

in Japan, 839

in Macau, 837-838

in Malaysia, 839-840

in Pakistan, 840

in the Philippines, 840

in Singapore, 840-841

in South Korea, 841

in Taiwan, 841-842 
in Thailand, 842

linguistic stereotypes, identity and, $771-772$

literature. See also Asian literatures, 787-811

Anglophone Asian literature, 787

Anglophone literature, 789

global English literature, 789

nationalist authors, 788

Pakistani English, 287-288

post-colonial, 788-789

postcolonial writing, 789

world Englishes literature, 801-806

world literature in English, 789

loanwords. See also lexical borrowing

Indian English, 257

MSEA (Mainland Southeast Asia), 37

LOB (Lancaster/Oslo-Bergen)

Corpus, 743

localization, case studies, 772-773

Girls Out Loud, 775-776

Love Psychedelico, 773-775

Macau, 529-546

the British and, 533

Cantonese, 530

Chinese languages in, 540-541

Civil Law, 871

code-switching, English/Cantonese, 530

Commercial Code, 536

contact varieties, 541-542

CPE (Chinese Pidgin English), 541

dialects, 530, 532

EMI higher education, 151, 156

English in Macau, 529-546

English in media, 535

English in schools, 121, 534-535

English users, 62

functions of English, 534-536

future of English, 542

history of English, 532-534

international business, 536

languages, 61, 530, 532

linguistic landscape studies, 837-838
Macau English versus English in Macau, 536-537

Maquista/Makista, 530

multilingualism, 537-542

origins, 532

Patuá Macaense, 530

Portuguese in, 538-539

SARs (Special Administrative Regions), 529

of China, 121

sociolinguistic context, 531-532

status of English, 534

tourism, international, 536

Macquarie dictionary, 217

Madurese, Sanskrit and, 40

Malay

Islam and, 34

Philippines and, 34

Proto-Malayic, 34

Singaporean speakers, 424

Tagalog and, 41

Malaysia, 51, 373-397

Bahasa Malaysia, 95

Common Law, 867-869

Education Ordinance, 375

EMI higher education, 143, 154

English in, 374-375

English in schools, 112-113

English users, 55

functions of English, 375-381

history of English, 373-374

increase of English, 714

language policies, 374-375

language-in-education policies, 94-95

languages, 55

linguistic landscape studies, 839-840

Mandarin, 375

National Language Act, 112

official language, 83

Razak Report of 1956, 375

Tamil, 375

Malaysian English, 373-397

ADR (Alternative Dispute

Resolution), 378

business, 376

code-switching, courts, 378 
Malaysian English (cont'd)

in education, 379-380

higher education, 380-381

grammar

noun-phrase structure, 386-389

verb phrase structure, 389-390

law, 376-377

law courts, 377-379

MBM-MBI (Memartabatkan Bahasa

Malaysia Mengukuhkan Bahasa Inggeris), 380

phonology

consonants, 384-385

suprasegmental features, 385-386

vowels, 382-384

UMSEP (University of Malaya's

Spoken English Project), 377-378

varieties, 179

vocabulary, 390-392

Malaysian English dictionaries, 225-226

Maldives

Common Law, 869-870

legal system English use, 870

Mandarin, Singaporean speakers, 424

The maneater of Malgudi (Narayan), 788

metaphors in religious language, 815

middle-class societies, growth, 765

Min languages, 35

minority languages, official language policy, 84-85

mixing, globalization and, 709

Mo, Timothy, 791

mobility, globalization and, 709

modernity, 4

Mongolian English dictionaries, 226

monolingual ideology, 82-83

APT (ASEAN+3), 82-84

morphology, 4

morphosyntactic features of Asian

Englishes, 169-170

Outer Circle varieties

Hong Kong English, 177-178

Indian English, 178-179

Malaysian English, 179

Philippine English, 180

Singapore English, 180-181 substrate language influences, 183-184

VUs (vernacular universals), 172-177

MSEA (Mainland Southeast Asia)

Austroasiatic languages, 22

Austroasiatic speakers, 23

Austronesian languages, 22

geography, 16

Hmong-Mien languages, 22, 23-24

Khasic languages, 18, 20

Kra-Dai languages, 22, 24-25

language contact and convergence, 30

lexical borrowing and, 37

linguistic convergence, 22

linguistic phyla, 22

Munda languages, 18, 20

political integration, 26-28

rice cultivation and, 22

Sino-Tibetan languages, 22, 24

Southeastern Riverine Hypothesis, 23

multilingualism, 4-5

ASEAN and, 725-727

Cambodia, 649-650

diversity of language families, 725

Hong Kong, 453-456

India, 245-247

Indonesia, 605-607

Laos, 668-670

linguistic landscapes, 833

Myanmar, 356-359

Pinoylish (Phillipines), 492

Singapore, 423-425

Sri Lanka, 345-347

superdiversity, 715-716

Taiwan, 548

Munda, 18, 20

MuPDAR (Multifactorial Prediction and Deviation Analysis with Regression), 753

Myanmar, 355-371

colonial era, 360

Common Law, 869-870

EfECT (English for Education College Trainers Project), 362-363

EMI higher education, 144, 154

English in schools, 113-114

English users, 55-56 
ethnic groups, 356, 358

history of English, 359-362

Independence Government, 360-361

language distribution, 358

languages, 55-56

legal system English use, 869

multilingualism, 356-359

official language, 83

regions, 357

religions, 356

Revolutionary Council

Government, 361

Socialist Government, 361

State Law and Restoration Council, 361-362

states, 357

union territory, 357

Myanmar English, 355-371

CESR (Comprehensive Education Sector Review), 362

discourse, 367

compliments and responses, 368 greetings, 368

English education, 362-363

grammar, phrases, 366, 367

lexis, 367

phonology

connected speech, 365

consonant clusters, 365

consonants, 364-365

diphthongs, 364

vowels, 364

Myanmar English dictionaries, 226

national languages, ethnic identity and, 793-794

nation-states, legal systems, 864

Negara Brunei Darussalam. See Brunei

Nehru, Jawaharlal, 788

Nepal, 317-336

Common Law, 869-870

ELT (English language teaching), 321

EMI higher education, 139-140, 154

English education, 319

English users, 56

history of English, 317-321

indigenous languages, 326
Khasa Bhasha, 318

Kirantis, 317-318

language and religion, 317

language hegemony, 320

languages, 56

legal system English use, 869-870

linguistic elitism in, 318

linguistic politics, 318

MLE (multilingual education), 327

multilingualism, 326-328

Nepali as state language, 318-319

NESP (New Education System Plan), 321

Newari, 318

Sanskrit, 317-318

Nepal English dictionaries, 226

Nepali English, 317-336

code-mixing, 328-329

code-switching, 328-329

constitution and, 321-322

education, MLE (multilingual education), 327

and English of British Raj, 323

features, 328-329

functions

creative, 325-326

instrumental, 324

interpersonal, 324-325

regulative, 324

globalization and, 325-326

language policies, 321-323

literature, 325

Nenglish, 329

neocolonialism and, 327-328

neologisms, 328

Nepalese English, 329

pluralization, 328

New Englishes, 171

NHEEE (National Higher Education

Entrance Examination),

China, 93

norms, 1

NOW (News on the Web), 747

Old Javanese, 39-40

Sanskrit and, 40 
Old Malay, Sanskrit and, 40

Orient, 796

Oriental, 796

orthographic reform, literacy increases and, 86

The other tongue: English across cultures (Kachru), 2

Outer Circle, 1, 4, 5-6

EMI higher education, 137, 153-155

East Asian societies, 141-146

South Asian societies, 138-141

Southeast Asian societies, 141-146

English in schools

Bangladesh, 108-109

Brunei, 109-110

Hong Kong, 110-112

India, 112

Malaysia, 112-113

Myanmar, 113-114

Nepal, 320-321

Pakistani, 114

Philippines, 114-115

Singapore, 115-116

Sri Lanka, 116

ESL (English as a Second Language), 107,171

substrates, 173

varieties

Hong Kong English, 177-179

Indian English, 178-179

Malaysian English, 179

Philippine English, 180

Singapore English, 180-181

WAMVE and, 174

Outer Circle Asian societies, 1-10, 243-500

Outer Circle Englishes, 243-500

Oxford English Dictionary (OED), 215

Pakistan, 51, 279-296

EMI higher education, 140, 154

English in schools, 114

English users, 56-57

functions of English, 281-282

history of English in, 280-281 language policies, 1973 Constitution, 281-282

languages, 56-57

linguistic landscape studies, 840

local language in legal system, 866

multilingualism, 280-283

status of English, 281-282

Pakistan English dictionaries, 226

Pakistani English, 279-296

culture and, 286-287

literature, $287-288$

education and, 288-289

features, 283-285

discourse, 285-286

future prospects, 290-292

grammatical features, 284-285

lexis, 284

phonology, 283-284

policies, 289-290

status and, 286

Papuan populations, Austronesians and, 30

paradigm shift in English studies, 1-2

Persian, 35-36

Philippine English, 176, 479-500

American English and, 195-196

BEP (Bilingual Education

Policy), 489

call centers, 490

competence, 488

creative writing, 482-483

endonormativity, 486

features, 481-489

grammatical features, 486

lexical properties, 482-483

lexical trends, 484-485

literature, 485

MTB-MLE (Mother Tongue-based

Multilingual Education), 490

Pinoylish, 479, 491-495

policies, 489-491

pronunciation model, 487

Standard Filipino English, 482

varieties, 180

Philippine English dictionaries, 227

Philippines, 51, 479-500

American colonialism, 480-481 
Anglophone literature, 793-795

Atkinson, Fred W., 480

BEP (Bilingual Education Policy), 95-96

colonial pluralism and, 795

cultural pluralism and, 793

EMI higher education, 144-145, 155

English in schools, 114-115

English users, 57

language-in-education policies, 95-97

languages, 57

linguistic landscape studies, 840

local language in legal system, 866-867

Malay and, 34

MTB-MLE (Mother Tongue-Based Multilingual Education), 96, 114-115

NLI (National Language Institute), 86 official language, 83

phonology, 195-196

Pinoylish, 491-495

Spanish colonial period, 480

US colonialism, 114-115

phonology, 4, 189-207

Brunei, 192

China, 197-198

Hong Kong, 192-193

India, 193-195

Japan, 198-199

Lankan English (Sri Lanka), 345

Philippines, 195-196

Singapore, 191

Vietnam, 199-200

pluralism English, 1, 3

policies

language choice, $88-90$

language education, 89

pedagogy, 92-93

programs for teaching language, 90-91

teacher qualifications, 91-92

popular culture, 763-764

bilingual creativity and, 769-771

decorative English, 769-770

identity and, 771-772

language mixing and, 769-771 middle-class societies, growth, 765

music, 772

population

agriculture and, 17-18

animal husbandry and, 17-18

postcolonial Englishes, 4

postcolonial literature, 789

postcolonial nation-states, 792

postcolonial studies, Asian writing and, 790-791

postcolonial theory, linguistics and, 218-219

proficiency levels, 65-72

Proto-Malayic, 34

Proto-Tibeto-Burman languages, 24

Putonghua, 54

QS (Quacquarelli Symonds) rankings, 157-159

queer diaspora in Asian literatures, 798-799

religion

dead languages, 817

divine languages, 817

as domain, 813

English and religion

Christianity and, $818-820$

Islam and, 820-822

as sacred and divine language, 816-817

IAL (international auxiliary language), Bahá'í faith, 817

linga francas and proselytization, 817-818

ritualized language, $814-815$

sacred languages, 817

spiritual intelligence, English and, 820-821

religious conversion, Indian influence, 40

religious language

divine languages, 817

English as sacred and divine, 816-817

metaphors, 815

sacred languages, 817 
religious law, 875

religious practice, 764

religious texts, Sanskrit, 31-34

risk, globalization and, 709, 720

ritualized language in religious practices, 814-815

Roy, Arundhati, 791

Rushdi, Salman, 791

sacred languages, 817

Sanskrit

Buddhism and, 33-34

Indonesian and, 39-40

Madurese and, 40

Old Javanese and, 40

Old Malay and, 40

religious texts, $31-34$

SAR (Special Administrative Region) in Hong Kong, 843

SAT (Scholastic Assessment Test), 510

SCE (Singapore Colloquial English), 180

Singlish, 191

semiotic landscapes, 834

geosemiotics, 835

Shanghai rankings, 157-159

shariah law, 875

Brunei, 865

Singapore, 865

Singapore, 51, 419-447

Chinese speakers, 424

Common Law, 865

current status of English, 428-429

EMI higher education, 145-146, 155

English education, 425-428

English in schools, 115-116

English schools, 427

English users, 57

functions of English, 428-429

future of English, 440-442

history of English, English education, 425-428

increase of English, 714

language planning, 428-429

language-in-education policies, 97-98

languages, 57

languages by ethnic group, 425 legal system, monolingual, 865

linguistic landscape studies, 840-841

Malay speakers, 424

Mandarin speakers, 424

multilingualism, 423-425

phonology, 191

population, 423-424

Speak Mandarin Campaign, 115

Tamil speakers, 424

Singapore Colloquial English, 422, 437-438

Singapore English, 176, 419-447

British English and, 420

CSE (Colloquial Singaporean English), 775-776

diglossia, 420

language attitude studies, 437-438

language identity and, 438-440

lexical features, 421

Mother Tongue policy, 429-431

expertise, 433

functions of English, 433-434

identity and, 435-436

parentless tongues, 436

SCE (Singapore Colloquial English), 180

Singlish, 419-420

SSE (Singapore Standard English), 180 standard, 420

syntactic features, 421

varieties, $180-181$

Singapore English dictionaries, 227-228

Singlish, 419-420

Sinitic, 34

Kra-Dai and, 37-38

Sinosphere, 27

Sino-Tibetan languages, 21

Macau, 61

MSEA, 24

Tai languages and, 37-38

Smith, Larry, World Englishes, 2, 367

sociolinguistics

Hong Kong English, 177

language and religion, 822-824

linguistic landscapes, 833

superdiversity, 715-716 


\section{South Asia}

Austroasiatic languages, 18-20

Dravidian languages, 20

EMI higher education, 138-141

geography, 15-16

Indo-Aryan languages, 20

Indo-Aryan Dravidian contact, 29-30

Indo-Gangetic language, 29

Kra-Dai speakers, 21-22

language contact and convergence, 29-30

linguistic epicentre, 176

major language families, 17-18

monsoonal patterns, 16

Sino-Tibetan languages, 21

Tibeto-Burman languages, 21

South Korea

ageism, 772

Civil Law, 870-871

Dongmunhak (Common Script Learning), 119

EMI higher education, 151-152, 156

English in, 588

English in schools, 119-120

English users, 62-63

languages, 61-62

linguistic landscape studies, 841

NEC (National English Curriculum), 120

Suneung (College Scholastic Ability Test), 120

Yukyonggongwon (Teaching English

Public Institute), 119-120

Southeast Asia

ASEAN, 725-740

colonial pluralism, 793

EMI higher education, 141-146

geography, 15-16

literature in English, 792-796

major language families, $17-18$

monsoonal patterns, 16

postcolonial nation-state and, 792

speed, globalization and, 709

Sprachräume, 752-753

Sri Lanka, 51, 337-353

code-mixing, 345-347
Common Law, 867-869

development of English, 337-341

education, English in, 340-341

EMI higher education, 141, 155

English in schools, 116

English users, 58

language policies colonial, 338-339

postcolonial, 339-340

languages, 58

multilingualism, 345-347

Muslim population, 338

Sinhala, 338

social class, English and, 341

SSLE (Standard Sri Lankan English), 341

Tamil, 338

Sri Lankan English, 337-353

British English, 342-343

emergence, 342

habitual speakers, 342

Lankan English code-mixing and, 345-347

code-mixing in popular song, 347-349

grammar, 343-345

lexis, 345

multilingualism and, 345-347

phonology, 345

popular music, 346

mixed language, 347

popular music, 346

Sri Lankan English dictionaries, 228-229

SSE (Singapore Standard English), 180, 191

standardization, globalization and, 709, 718-719

structural borrowing, language contact and, 28-29

structural convergence, language contact and, 28-29

substrate languages, influence, 183-184

superdiversity, 715-716, 835

code switching and, 716-717

India, 716-717

syntax, 4, 169-188 
Tagalog

Malay and, 41

Spanish loanwords, 41

Taiwan, 547-567

code-switching, 549

DPP (Democratic Progressive Party), 548-549

EMI higher education, 152-153, 157

English in schools, 121-122

English in Taiwan, 547-567

English users, 63

ethnolinguistic groups, 547

Hakka people, 548

history of English, 549-553

Hokkien people, 547-548

KMT (Kuomintang) government, 548

language policy, 548

languages, 63

linguistic landscape studies, 841-842

Mandarin-Hokkien code-switching, 549

Mandarinization, 548

multilingualism, 548

pseudo-monolingualism, 549

Taiwanese English

American English, 560-562

BEPs (bilingual English programs), 555

code-mixing, 559-560

cultural aspects, 559-560

elementary education, 555

future of, 563-564

lexis, 558-559

in media, 556

in medicine, 555-556

phonology, 557-558

secondary education, 555

as semi-official language, 553-554

syntax, 559

tertiary education, 554-555

TOEIC (Test of English for

International Communication), 555

Twin Peaks phenomenon, 562-564

Taiwanese English dictionaries, 229

Tamil, Singaporean speakers, 424

teacher qualifications, 91-92
TESOL (Teaching English to speakers of other languages), English, Christianity and, 818-820

Thai English

cultural aspects, 640-641

discourse features, 639-640

education, compulsory, 633

EMI (English-medium instruction), 642

functions, 634-635

higher education, 635

King Rama IV, 632-633

language education, 641-643

lexis, 637-638

phonology, 636-637

status, 634-635

syntactic features, 638-639

varieties, 183

Thai English dictionaries, 229

Thailand, 51, 629-648

absolutism, 633-634

Basic Education Core Curriculum of 2008,122

Civil Law, 871-872

ELT (English language teaching), 634

EMI higher education, 148, 157

English in schools, 122

English in Thailand, 629-648

English proficiency, 630

English users, 63-64

ethnic groups, 631

history of English, 632-634

languages, 63-64

linguistic landscape studies, 842

National Education Commission, 634

NEA (National Education Act), 122

social mobility and, 632

sociolinguistics, 630

THE (Times Higher Education) World

University Rankings, 157-159

Three Circles model, 1, 107, 169, 802

early controversies, 189-190

Expanding Circle, 1, 5-6, 713

globalization and, 713

Inner Circle, 1, 713

mixing, 715-718

mobility, 715-718

Outer Circle, 1, 5-6, 713 
Tibeto-Burman languages, 21

Austroasiatic languages and, 23

Timor Leste, Civil Law, 873

TOEFL (Test of English as a Foreign

Language), 50, 510

proficiency levels, 65-69

translingual practices, $717-718$

trilingualism

Hong Kong, 111

India, 112

urban language, 4

urbanization, 4

Vietnam, 51, 683-703

Austroasiatic languages, 684

CEFR, 697

Civil Law, 872

EMI higher education, 148-149, 157

English in schools, 123

English in Vietnam, 683-703

English users, 64

French colonialism, 123, 684

history of English, 684-685

language-in-education policies, 98-99

languages, 64

minority groups, 683

Mon-Khmer languages, 684

National Foreign Language 2020

Project, 696

phonology, 199-200

post-war economy, 685

Russian language, 123
Vietnamese

as institutional language, 683

writing system, 34

Vietnamese English

in business, 686

communication and, 686

culture and, 695-696

in education, 686-688

education and, 696-698

EMI (English-medium instruction), 688-689

future of, 698-699

language policies, 696-698

phonology/phonetics

consonants, 690-691

grammar, 692-694

tone, 691-692

vocabulary, 694

vowels, 690

Vietnamese society and, 689

Vietnamese English dictionaries, 229

vocabulary, 4, 209-240

VOICE (Vienna-Oxford International Corpus of English), 731, 748

VUs (vernacular universals), 172-177

WAMVE (World Atlas of Morphosyntactic Variation in English), 172-174

WE (world Englishes), 1-10

world Englishes. See WE (world

Englishes)

World Englishes, 2

world Englishes literature, 787-811 
